Adimurthi · Frédéric Robert · Michael Struwe

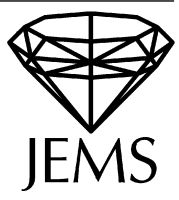

\title{
Concentration phenomena for Liouville's equation in dimension four
}

Received June 6, 2005

\section{Introduction}

Let $\Omega$ be a bounded domain of $\mathbb{R}^{4}$ and let $u_{k}$ be solutions to the equation

$$
\Delta^{2} u_{k}=V_{k} e^{4 u_{k}} \quad \text { in } \Omega,
$$

where

$$
V_{k} \rightarrow 1 \text { uniformly in } \Omega \text {, }
$$

as $k \rightarrow \infty$. Throughout the paper we denote as $\Delta=-\sum_{i}\left(\partial / \partial x^{i}\right)^{2}$ the Laplacian with the geometers' sign convention. Continuing the analysis of [19], here we study the compactness properties of equation (1).

Equation (1) is the fourth order analogue of Liouville's equation. Thus, for problem (1), 2] we may expect similar results to those obtained by Brézis-Merle [3] in the two-dimensional case. Recall the following result from [3] and its improvement by LiShafrir [11].

Theorem 1.1. Let $\Sigma$ be a bounded domain of $\mathbb{R}^{2}$ and let $\left(u_{k}\right)_{k \in \mathbb{N}}$ be a sequence of solutions to the equation

$$
\Delta u_{k}=V_{k} e^{2 u_{k}} \quad \text { in } \Sigma,
$$

where $V_{k} \rightarrow 1$ uniformly in $\Sigma$ as $k \rightarrow \infty$, and satisfying the uniform bound

$$
\int_{\Sigma} V_{k} e^{2 u_{k}} d x \leq \Lambda
$$

for some $\Lambda>0$. Then either

(i) $\left(u_{k}\right)_{k \in \mathbb{N}}$ is locally bounded in $C^{1, \alpha}$ on $\Sigma$ for every $\alpha<1$, or

(ii) there exists a subsequence $K \subset \mathbb{N}$ such that $u_{k} \rightarrow-\infty$ locally uniformly in $\Omega$ as $k \rightarrow \infty, k \in K$, or

Adimurthi: TIFR Centre, Indian Institute of Science, Bangalore, 560 012, India; e-mail: aditi@math.tifrbng.res.in

F. Robert: Université de Nice-Sophia Antipolis, Laboratoire J. A. Dieudonné, Parc Valrose, 06108 Nice Cedex 2, France; e-mail: frobert@ math.unice.fr

M. Struwe: Mathematik, ETH Zürich, CH-8092 Zürich; e-mail: michael.struwe@ math.ethz.ch 
(iii) there exist a subsequence $K \subset \mathbb{N}$ and at most finitely many points $x^{(i)} \in \Omega, 1 \leq i \leq I$, with corresponding numbers $\beta_{i} \in 4 \pi \mathbb{N}$ such that $V_{k} e^{2 u_{k}} d x \rightarrow \sum_{i=1}^{I} \beta_{i} \delta_{x^{(i)}}$ weakly in the sense of measures while $u_{k} \rightarrow-\infty$ locally uniformly in $\Omega \backslash\left\{x^{(i)} ; 1 \leq i \leq I\right\}$ when $k \rightarrow \infty, k \in K$.

Moreover, near any concentration point $x^{(i)}$, after rescaling

$$
v_{k}(x)=u_{k}\left(x_{k}+r_{k} x\right)+\log r_{k}, \quad W_{k}(x)=V_{k}\left(x_{k}+r_{k} x\right)
$$

with suitable sequences $x_{k} \rightarrow x^{(i)}$ and $r_{k} \rightarrow 0$ as $k \rightarrow \infty$, a subsequence satisfies $v_{k} \rightarrow v$ locally uniformly in $C^{1, \alpha}$ on $\mathbb{R}^{2}$, where $v$ is a solution of Liouville's equation

$$
\Delta u=e^{2 u} \quad \text { on } \mathbb{R}^{2} \quad \text { with } \quad \int_{\mathbb{R}^{2}} e^{2 u} d x<\infty .
$$

Geometrically speaking, the solutions $u_{k}$ to equation (3) correspond to conformal metrics $g_{k}=e^{2 u_{k}} g_{\mathbb{R}^{2}}$ on $\Sigma$ with Gauss curvature $V_{k}$. The fact that all solutions $u$ of equation (6) by a result of Chen-Li [5] are induced by conformal metrics $e^{2 u} g_{\mathbb{R}^{2}}$ on $\mathbb{R}^{2}$ that are obtained by stereographic projection of the standard sphere then gives rise to the observed quantization. Multiple blow-up at a point is possible, as shown by X. Chen [6].

Similarly, the solutions $u_{k}$ to (1) induce conformal metrics $g_{k}=e^{2 u_{k}} g_{\mathbb{R}^{4}}$ on $\Omega$ having $Q$-curvature proportional to $V_{k}$. In contrast to the two-dimensional case, however, there is a much greater abundance of solutions to the corresponding limit equation

$$
\Delta^{2} u=e^{4 u} \quad \text { on } \mathbb{R}^{4} .
$$

In fact, by a result of Chang-Chen [4] for any $\alpha \in] 0,16 \pi^{2}$ ] there exists a solution $u_{\alpha}$ of (7) of total volume $\int_{\mathbb{R}^{4}} e^{4 u_{\alpha}} d x=\alpha$ which for $\alpha<16 \pi^{2}$ fundamentally differs from the solution $u(x)=\log \left(\sqrt{96} /\left(\sqrt{96}+|x|^{2}\right)\right)$ corresponding to the metric obtained by pullback of the spherical metric on $S^{4}$ under stereographic projection. Only the latter solution (and any solution obtained from $u$ by rescaling as in (5) ) achieves the maximal value $\int_{\mathbb{R}^{4}} e^{4 u} d x=16 \pi^{2}$. If we then consider a suitable sequence $u_{k}=u_{\alpha_{k}}$ with $\alpha_{k} \rightarrow 0$ as $k \rightarrow \infty$, normalized as in (5) so that $u_{k} \leq u_{k}(0)=k$, we can even achieve that $\left(u_{k}\right)_{k \in \mathbb{N}}$ blows up at $x^{(1)}=0$ in the sense that $u_{k}(0) \rightarrow \infty$ while $u_{k}(x) \rightarrow-\infty$ for any $x \neq 0$ as $k \rightarrow \infty$.

As shown in Example 3.1, solutions to equation (1) with a similar concentration behavior exist even in the radially symmetric case.

There is a further complication in the four-dimensional case, illustrated by the following simple example. Consider the sequence $\left(v_{k}\right)$ on $\mathbb{R}^{4}$, defined by letting $v_{k}(x)=$ $w_{k}\left(\left|x^{1}\right|\right)$, where for $k \in \mathbb{N}$ we let $w_{k}$ solve the initial value problem for the ordinary differential equation $w_{k}^{\prime \prime \prime \prime}=e^{4 w_{k}}$ on $0<s<\infty$ with initial data $w_{k}(0)=w_{k}^{\prime}(0)=$ $w_{k}^{\prime \prime \prime}(0)=0, w_{k}^{\prime \prime}(0)=-k$. Given $\Lambda>0$, we can then find a sequence of radii $R_{k}>0$ such that $\int_{B_{R_{k}}(0)} e^{4 v_{k}} d x=\Lambda$. Observe that $R_{k} \rightarrow \infty$ as $k \rightarrow \infty$. Scaling as in (5), we then obtain a sequence of solutions $u_{k}(x)=v_{k}\left(R_{k} x\right)+\log R_{k}$ to (7) on $\Omega=B_{1}(0)$ such that $u_{k}(x) \rightarrow \infty$ for all $x \in S_{0}=\left\{x \in \Omega ; x^{1}=0\right\}$ and $u_{k} \rightarrow-\infty$ away from $S_{0}$ as $k \rightarrow \infty$. Scaling back as in (5), from $\left(u_{k}\right)$ we recover the normalized functions $v_{k}$ which 
fail to converge to a solution of the limit problem (7) and develop an interior layer on the hypersurface $\left\{x \in \mathbb{R}^{4} ; x^{1}=0\right\}$ instead.

These comments illustrate that conclusions (i), (ii) and (iii) of Theorem 1.1 do not exhaustively describe all the possible concentration phenomena for (1). In fact, the following concentration-compactness result seems best possible.

Theorem 1.2. Let $\Omega$ be a bounded domain of $\mathbb{R}^{4}$ and let $\left(u_{k}\right)_{k \in \mathbb{N}}$ be a sequence of solutions to (1), (2) above. Assume that there exists $\Lambda>0$ such that

$$
\int_{\Omega} V_{k} e^{4 u_{k}} d x \leq \Lambda
$$

for all $k$. Then either

(i) a subsequence $\left(u_{k}\right)$ is relatively compact in $C_{\mathrm{loc}}^{3, \alpha}(\Omega)$, or

(ii) there exist a subsequence $\left(u_{k}\right)$ and a closed nowhere dense set $S_{0}$ of vanishing measure and at most finitely many points $x^{(i)} \in \Omega, 1 \leq i \leq I \leq C \Lambda$, such that, letting

$$
S=S_{0} \cup\left\{x^{(i)} ; 1 \leq i \leq I\right\}
$$

we have $u_{k} \rightarrow-\infty$ locally uniformly away from $S$ as $k \rightarrow \infty$.

Moreover, there is a sequence of numbers $\beta_{k} \rightarrow \infty$ such that

$$
u_{k} / \beta_{k} \rightarrow \varphi \quad \text { in } C_{\mathrm{loc}}^{3, \alpha}(\Omega \backslash S),
$$

where $\varphi \in C^{4}\left(\Omega \backslash\left\{x^{(i)} ; 1 \leq i \leq I\right\}\right)$ is such that

$$
\Delta^{2} \varphi=0, \quad \varphi \leq 0, \quad \varphi \neq 0,
$$

and

$$
S_{0}=\left\{x \in \Omega \backslash\left\{x^{(i)} ; 1 \leq i \leq I\right\} ; \varphi(x)=0\right\} .
$$

Finally, near any point $x_{0} \in S$ where $\sup _{B_{r}\left(x_{0}\right)} u_{k} \rightarrow \infty$ for every $r>0$ as $k \rightarrow \infty$, in particular, near any concentration point $x^{(i)}$, there exist points $x_{k} \rightarrow x_{0}$, numbers $L_{k} \rightarrow \infty$, and suitable radii $r_{k} \rightarrow 0$ such that after normalizing we have

$$
v_{k}(x)=u_{k}\left(x_{k}+r_{k} x\right)+\log r_{k} \leq 0 \leq \log 2+v_{k}(0) \quad \text { for }|x| \leq L_{k} .
$$

As $k \rightarrow \infty$ then either a subsequence $v_{k} \rightarrow v$ in $C_{\text {loc }}^{3, \alpha}\left(\mathbb{R}^{4}\right)$, where $v$ solves the limit equation (7), or $v_{k} \rightarrow-\infty$ almost everywhere and there is a sequence of numbers $\gamma_{k} \rightarrow \infty$ such that a subsequence satisfies

$$
v_{k} / \gamma_{k} \rightarrow \psi \quad \text { in } C_{\mathrm{loc}}^{3, \alpha}\left(\mathbb{R}^{4}\right),
$$

where $\psi \leq 0$ is a non-constant quadratic polynomial. 
We regard Theorem 1.2 as a first step towards a more complete description of the possible concentration behavior of sequences of solutions to problem (1), (2).

Considering (1) as a system of second order equations for $u_{k}$ and $\Delta u_{k}$, respectively, it is possible to obtain some partial results in this regard from the observation that (1), (2) provide uniform integral bounds for $\Delta u_{k}$ up to a remainder given by a harmonic function. The latter component may be controlled if one imposes, for instance, the Navier boundary conditions $u_{k}=\Delta u_{k}=0$ on $\partial \Omega$. In fact, in this case, assuming that each $V_{k}$ is a constant $\lambda_{k}>0$ that tends to 0 as $k \rightarrow \infty$, J. Wei [21] has shown (in the notation of Theorem 1.2) that $S_{0}=\emptyset$ and that at any concentration point $x^{(i)}$ suitably rescaled functions satisfy $v_{k} \rightarrow v$ in $C_{\text {loc }}^{3, \alpha}\left(\mathbb{R}^{4}\right)$, where $v$ is the profile induced by stereographic projection.

As shown by Robert [18], the same result holds if for some open subset $\emptyset \neq \omega \subset \Omega$ we have the a priori bounds

$$
\left\|\left(\Delta u_{k}\right)^{-}\right\|_{L^{1}(\Omega)} \leq C, \quad\left\|\left(\Delta u_{k}\right)^{+}\right\|_{L^{1}(\omega)} \leq C,
$$

for all $k \in \mathbb{N}$, where $s^{ \pm}= \pm \max \{0, \pm s\}$. Also in the radially symmetric case there is a complete description of the possible concentration patterns; see [18].

In the geometric context similar results hold for the related problem of describing the possible concentration behavior of solutions to the equation of prescribed $Q$-curvature on a closed 4-manifold $M$. Here the bi-Laplacian in equation (1) is replaced by the Paneitz-Branson operator and $V_{k}$ may again be interpreted as being proportional to the $Q$-curvature of the metric $g_{k}=e^{2 u_{k}} g_{M}$. In the case when $M=S^{4}$, Malchiodi-Struwe [14] have shown that any such sequence $\left(g_{k}\right)$ of metrics when $V_{k} \rightarrow 1$ uniformly either is relatively compact or blows up at a single concentration point where a round spherical metric forms after rescaling. Further compactness results and references can be found in the papers of Druet-Robert [8] and Malchiodi [13].

Related results on compactness issues for fourth order equations can be found in Hebey-Robert-Wen [10], C. S. Lin [12] and Robert [17]; concentration-compactness issues for problems with exponential nonlinearities in two dimensions have been treated in Adimurthi-Druet [1], Adimurthi-Struwe [2] and Druet [7].

In the following the letter $C$ denotes a generic constant independent of $k$ which may change from line to line and even within the same line.

\section{Proof of Theorem 1.2}

Recall the following result, obtained independently by C. S. Lin [12, Lemma 2.3] and J. Wei [21, Lemma 2.3], which generalizes Theorem 1 from [3] to higher dimensions.

Theorem 2.1. Let $v$ be a solution to the equation

$$
\Delta^{2} v=f \quad \text { in } B_{R}\left(x_{0}\right) \subset \mathbb{R}^{4}
$$

with

$$
v=\Delta v=0 \quad \text { on } \partial B_{R}\left(x_{0}\right),
$$


where $f \in L^{1}\left(B_{R}\left(x_{0}\right)\right)$ satisfies

$$
\|f\|_{L^{1}}=\alpha<8 \pi^{2} .
$$

Then for any $p<8 \pi^{2} / \alpha$ we have $e^{4 p|v|} \in L^{1}\left(B_{R}\left(x_{0}\right)\right)$ with

$$
\int_{B_{R}\left(x_{0}\right)} e^{4 p|v|} d x \leq C(p) R^{4} .
$$

The following characterization of biharmonic functions, due to Pizzetti [16], can be found in [15]. Denote by $f_{B_{R}(y)} h d x$ the average of $h$ over $B_{R}(y)$, etc.

Lemma 2.2. For any $n \in \mathbb{N}$, any solution $h$ of

$$
\Delta^{2} h=0 \quad \text { in } B_{R}(y) \subset \mathbb{R}^{n}
$$

satisfies

$$
h(y)-f_{B_{R}(y)} h(z) d z=\frac{R^{2}}{2(n+2)} \Delta h(y) .
$$

Proof. For convenience, we indicate a short proof. We may assume that $B_{R}(y)=B_{R}(0)$ $=B_{R}$. For $0<r<R$ let $G_{r}$ be the fundamental solution of the operator $\Delta^{2}$ on $B_{r}$ satisfying $G_{r}=\Delta G_{r}=0$ on $\partial B_{r}$. Note that $G_{r}(x)=r^{4-n} G_{1}(x / r)$. (If $n=4$, we have $G_{r}(x)=c_{0}\left(\log \frac{r}{|x|}-\frac{r^{2}-|x|^{2}}{4 r^{2}}\right)$.) Applying the mean value formula to the harmonic function $\Delta h$, for some constants $c_{1}, c_{2}$ we have

$$
\begin{aligned}
0 & =\int_{B_{r}} G_{r} \Delta^{2} h d x=h(0)+\int_{\partial B_{r}}\left(\frac{\partial}{\partial n} G_{r} \Delta h+\frac{\partial}{\partial n} \Delta G_{r} h\right) d o \\
& =h(0)-f_{\partial B_{r}}\left(c_{1} r^{2} \Delta h+c_{2} h\right) d o=h(0)-c_{1} r^{2} \Delta h(0)-c_{2} f_{\partial B_{r}} h d o ;
\end{aligned}
$$

that is, for some constants $c_{3}, c_{4}$,

$$
n r^{n-1} h(0)=c_{3} r^{n+1} \Delta h(0)+c_{4} \int_{\partial B_{r}} h d o .
$$

Integrating over $0<r<R$ and dividing by $R^{n}$, we obtain the identity

$$
h(0)=c_{5} R^{2} \Delta h(0)+c_{6} f_{B_{R}} h d x
$$

with uniform constants $c_{5}, c_{6}$ for all biharmonic functions $h$ on $B_{R}$. Inserting a harmonic function $h$, we obtain the value $c_{6}=1$, whereas the choice $h(x)=|x|^{2}$ yields $c_{5}=$ $1 / 2(n+2)$.

Lemma2.2 gives rise to a Liouville property for biharmonic functions on $\mathbb{R}^{n}$. To see this first recall the following result for harmonic functions. 
Theorem 2.3. Suppose that the function $H$ is harmonic on $\mathbb{R}^{n}$ with $H(x) \leq C\left(1+|x|^{l}\right)$ for some $l \in \mathbb{N}$. Then $d^{l+1} H \equiv 0$; that is, $H$ is a polynomial of degree at most $l$.

Proof. From the mean value property of the harmonic function $d^{l+1} H$, where $d^{k}$ now denotes any partial derivative of order $k$, for any $x$ and $R>0$ we have

$$
\left|d^{l+1} H(x)\right| \leq C R^{-(l+1)} f_{B_{R}(x)}|H(y)| d y ;
$$

see for instance Evans [9. Theorem 2.2.7, p. 29]. But if we assume $H(x) \leq C\left(1+|x|^{l}\right)$, the right hand side by the mean value poperty of $H$ up to an error of order $R^{-1}$ and up to a multiplicative constant equals $R^{-(l+1)} H(x)$, and the latter tends to 0 as $R \rightarrow \infty$ for any fixed $x$.

Together with Lemma 2.2 we now obtain the following result.

Theorem 2.4. Suppose that the function $h$ is biharmonic on $\mathbb{R}^{n}$ with $h(x) \leq C(1+|x|)$ for some $C \in \mathbb{R}$. Then $\Delta h \equiv$ const $\geq 0$ and $h$ is a polynomial of degree $\leq 2$.

Proof. From Lemma 2.2 and the assumption $h(y) \leq C(1+|y|)$ we obtain the equation

$$
\begin{aligned}
\Delta h(x) & =2(n+2) \lim _{R \rightarrow \infty} R^{-2} f_{B_{R}(x)}|h(y)| d y \\
& =2(n+2) \lim _{R \rightarrow \infty} R^{-2} f_{B_{R}(0)}|h(y)| d y=\Delta h(0)=: 2 n a
\end{aligned}
$$

for every $x \in \mathbb{R}^{n}$, where $a \geq 0$. The function $H(x)=h(x)+a|x|^{2}$ is then harmonic with $H(x) \leq C\left(1+|x|^{2}\right)$ and the claim follows from Theorem 2.3 .

Proof of Theorem 1.2 Choose a subsequence $k \rightarrow \infty$ and a maximal number of points $x^{(i)} \in \Omega, 1 \leq i \leq I$, such that for each $i$ and any $R>0$,

$$
\liminf _{k \rightarrow \infty} \int_{B_{R}\left(x^{(i)}\right)} V_{k} e^{4 u_{k}} d x \geq 8 \pi^{2} .
$$

By (8) we then have $I \leq C \Lambda$. Moreover, given $x_{0} \in \Omega \backslash\left\{x^{(i)} ; 1 \leq i \leq I\right\}$ ), we can choose a radius $R>0$ such that

$$
\limsup _{k \rightarrow \infty} \int_{B_{R}\left(x_{0}\right)} V_{k} e^{4 u_{k}} d x<8 \pi^{2} .
$$

For such $x_{0}$ and $R>0$ decompose

$$
u_{k}=v_{k}+h_{k} \quad \text { on } B_{R}\left(x_{0}\right),
$$

where $v_{k}$ satisfies

$$
\Delta^{2} v_{k}=V_{k} e^{4 u_{k}} \quad \text { in } B_{R}\left(x_{0}\right), \quad v_{k}=\Delta v_{k}=0 \quad \text { on } \partial B_{R}\left(x_{0}\right),
$$

and with $\Delta^{2} h_{k}=0$ in $B_{R}\left(x_{0}\right)$. 
By (8) and Theorem 2.1 we then have

$$
\left\|h_{k}^{+}\right\|_{L^{1}\left(B_{R}\left(x_{0}\right)\right)} \leq\left\|u_{k}^{+}\right\|_{L^{1}\left(B_{R}\left(x_{0}\right)\right)}+\left\|v_{k}\right\|_{L^{1}\left(B_{R}\left(x_{0}\right)\right)} \leq C,
$$

uniformly in $k$.

We now distinguish the following cases.

Case 1: Suppose that $\left\|h_{k}\right\|_{L^{1}\left(B_{R / 2}\left(x_{0}\right)\right)} \leq C$, uniformly in $k$. Then Lemma 2.2 shows that for all $x \in B_{R / 8}\left(x_{0}\right)$ we can bound

$$
\left|\Delta h_{k}(x)\right|=\left|f_{B_{R / 8}(x)} \Delta h_{k}(y) d y\right| \leq C R^{-2} f_{B_{R / 2}\left(x_{0}\right)}|h(z)| d z \leq C,
$$

uniformly in $k$ and $x$, and $\left(h_{k}\right)$ is locally bounded in $C^{4}$ on $B_{R / 8}\left(x_{0}\right)$. But then from Lemma 2.2 and (17) we also obtain

$$
f_{B_{R}\left(x_{0}\right)}|h(x)| d x \leq C-f_{B_{R}\left(x_{0}\right)} h(x) d x=C+\frac{1}{12} R^{2} \Delta h_{k}\left(x_{0}\right)-h_{k}\left(x_{0}\right) \leq C .
$$

By repeating the first step of the argument on any ball contained in $B_{R}\left(x_{0}\right)$ we then infer that $\left(h_{k}\right)$ is locally bounded in $C^{4}$ on $B_{R}\left(x_{0}\right)$.

But then by Theorem 2.1 and 16 we see that

$$
\Delta^{2} v_{k}=V_{k} e^{4 u_{k}}=\left(V_{k} e^{4 h_{k}}\right) e^{4 v_{k}}
$$

is locally bounded in $L^{p}$ on $B_{R}\left(x_{0}\right)$ for some uniform number $p>1$. Since Theorem 2.1 also yields uniform $L^{1}$-bounds for $v_{k}$, we may conclude that $\left(v_{k}\right)$ is locally bounded in $C^{3, \alpha}$ on $B_{R}\left(x_{0}\right)$ for any $\alpha<1$, and hence so is $\left(u_{k}\right)$.

Case 2: Now assume that $\beta_{k}:=\left\|h_{k}\right\|_{L^{1}\left(B_{R / 2}\left(x_{0}\right)\right)} \rightarrow \infty$ as $k \rightarrow \infty$. Normalize

$$
\varphi_{k}=\frac{h_{k}}{\left\|h_{k}\right\|_{L^{1}\left(B_{R / 2}\left(x_{0}\right)\right)}},
$$

so that $\left\|\varphi_{k}\right\|_{L^{1}\left(B_{R / 2}\left(x_{0}\right)\right)}=1$ for all $k$. By arguing as in Case 1 , we then find that $\left(\varphi_{k}\right)$ is locally bounded in $C^{4}$ on $B_{R}\left(x_{0}\right)$. A subsequence as $k \rightarrow \infty$ therefore converges in $C_{\mathrm{loc}}^{3, \alpha}\left(B_{R}\left(x_{0}\right)\right)$ to a limit $\varphi$ satisfying the equation $\Delta^{2} \varphi=0$ in $B_{R}\left(x_{0}\right)$ and with $\|\varphi\|_{L^{1}\left(B_{R / 2}\left(x_{0}\right)\right)}=1$. Clearly, the function $\varphi$ then cannot vanish identically. By (17), moreover, we have $\left\|\varphi^{+}\right\|_{L^{1}\left(B_{R}\left(x_{0}\right)\right)}=0$, and therefore $\varphi \leq 0$. It then follows from Lemma 2.2 that $\Delta \varphi(x) \neq 0$ at any point $x$ where $\varphi(x)=0$. The set $S_{0}=\left\{x \in B_{R}\left(x_{0}\right) ; \varphi(x)=0\right\}$ is hence of codimension $\geq 1$ and therefore also has vanishing measure; moreover, $S_{0}$ is closed and nowhere dense. Thus, we conclude that $\varphi<0$ almost everywhere and hence $h_{k}=\beta_{k} \varphi_{k} \rightarrow-\infty$ almost everywhere and locally uniformly away from $S_{0}$ as $k \rightarrow \infty$. Again observing that

$$
\Delta^{2} v_{k}=V_{k} e^{4 u_{k}}=\left(V_{k} e^{4 h_{k}}\right) e^{4 v_{k}}
$$

is locally bounded in $L^{p}$ on $B_{R}\left(x_{0}\right) \backslash S_{0}$ for some uniform number $p>1$, as before we conclude that $\left(v_{k}\right)$ is locally bounded in $C^{3, \alpha}$ for any $\alpha<1$ on $B_{R}\left(x_{0}\right) \backslash S_{0}$. It follows 
that $u_{k}=v_{k}+h_{k} \rightarrow-\infty$ almost everywhere and locally uniformly away from $S_{0}$ as $k \rightarrow \infty$ and $u_{k} / \beta_{k} \rightarrow \varphi$.

Since Cases 1 and 2 are mutually exclusive and since the region $\Omega \backslash\left\{x^{(i)} ; 1 \leq i \leq I\right\}$ is connected, upon covering this region with balls $B_{R}\left(x_{0}\right)$ as above we see that either a subsequence $\left(u_{k}\right)$ is locally bounded in $C^{3, \alpha}$ away from $\left\{x^{(i)} ; 1 \leq i \leq I\right\}$ for any $\alpha<1$, and hence $\left(u_{k}\right)$ is relatively compact in $C^{3, \alpha}$ on this domain for any $\alpha<1$, or $u_{k} \rightarrow-\infty$ almost everywhere and locally uniformly away from $S=S_{0} \cup\left\{x^{(i)} ; 1 \leq i \leq I\right\}$ ), with $\left(u_{k} / \beta_{k}\right)$ converging to a nontrivial biharmonic limit $\varphi \leq 0$.

Finally, we show that whenever there is concentration only the second case can occur, that is, $u_{k} \rightarrow-\infty$ almost everywhere as $k \rightarrow \infty$ if $\left.\left\{x^{(i)} ; 1 \leq i \leq I\right\}\right) \neq \emptyset$. Indeed, suppose by contradiction that there is at least one concentration point and that $u_{k} \rightarrow u$ in $C_{\text {loc }}^{3, \alpha}\left(\Omega \backslash\left\{x^{(i)} ; 1 \leq i \leq I\right\}\right)$ as $k \rightarrow \infty$. By Robert's result [18], or by the reasoning of Wei [21] we then have the convergence

$$
V_{k} e^{4 u_{k}} d x \rightarrow e^{4 u} d x+\sum_{i=1}^{I} m_{i} \delta_{x^{(i)}}
$$

weakly in the sense of measures, where $m_{i} \geq 16 \pi^{2}, 1 \leq i \leq I$. But near each $x^{(i)}$ the leading term in the Green function $G$ for the bi-Laplacian is given by

$$
G(x)=\frac{1}{8 \pi^{2}} \log \left(\frac{1}{\left|x-x^{(i)}\right|}\right) .
$$

By arguing as in Brézis-Merle [3, p. 1242 f.], we then conclude that

$$
u(x) \geq 2 \log \left(\frac{1}{\left|x-x^{(i)}\right|}\right)-C
$$

near $x^{(i)}$, and with a constant $c_{0}>0$ we find

$$
e^{4 u(x)} \geq c_{0}\left|x-x^{(i)}\right|^{-8} \notin L^{1}(\Omega),
$$

thus contradicting the hypothesis $(8)$. This completes the proof of the asserted macroscopic concentration behavior of $\left(u_{k}\right)$.

In order to analyze the asymptotic behavior of $\left(u_{k}\right)$ near concentration points we adapt an argument of Schoen to our setting; see [20, proof of Theorem 2.2]. Let $x_{0} \in S$ with $\sup _{B_{r}\left(x_{0}\right)} u_{k} \rightarrow \infty$ for every $r>0$ as $k \rightarrow \infty$. For $r \geq 0$ denote as $K_{r}\left(x_{0}\right)=\{x$; $\left.\left|x-x_{0}\right| \leq r\right\}$ the closed $r$-ball centered at $x_{0}$. For $R<\operatorname{dist}\left(x_{0}, \partial \Omega\right)$ choose $0 \leq r_{k}<R$ and $x_{k} \in K_{r_{k}}\left(x_{0}\right)$ such that

$$
\left(R-r_{k}\right) e^{u_{k}\left(x_{k}\right)}=\left(R-r_{k}\right) \sup _{K_{r_{k}}\left(x_{0}\right)} e^{u_{k}}=\max _{0 \leq r<R}\left((R-r) \sup _{K_{r}\left(x_{0}\right)} e^{u_{k}}\right)=: L_{k} .
$$

Note that $L_{k} \rightarrow \infty$ as $k \rightarrow \infty$. Define $s_{k}=\left(R-r_{k}\right) / 2 L_{k}$ and similar to (5) let

$$
v_{k}(x)=u_{k}\left(x_{k}+s_{k} x\right)+\log s_{k},
$$


satisfying

$$
\begin{aligned}
& \sup _{K_{L_{k}}(0)} e^{v_{k}}=s_{k} \sup _{K_{\left(R-r_{k}\right) / 2}\left(x_{k}\right)} e^{u_{k}} \leq s_{k} \sup _{K_{\left(R+r_{k}\right) / 2\left(x_{0}\right)}} e^{u_{k}}=L_{k}^{-1}\left(R-\frac{R+r_{k}}{2}\right) \sup _{K_{\left(R+r_{k}\right) / 2}\left(x_{0}\right)} e^{u_{k}} \\
& \leq L_{k}^{-1}\left(R-r_{k}\right) e^{u_{k}\left(x_{k}\right)}=1=2 e^{v_{k}(0)}
\end{aligned}
$$

in view of (18), which is equivalent to the assertion (9).

Observe that $v_{k}$ solves the equation

$$
\Delta^{2} v_{k}=W_{k} e^{4 v_{k}}
$$

in $B_{L_{k}}(0)$, where the sequence of balls $B_{L_{k}}(0)$ exhausts all of $\mathbb{R}^{4}$ and

$$
W_{k}(x)=V_{k}\left(x_{k}+s_{k} x\right) \rightarrow 1 \quad \text { locally uniformly in } \mathbb{R}^{4} ;
$$

moreover,

$$
\int_{B_{L_{k}}(0)} W_{k} e^{4 v_{k}} d x \leq \Lambda
$$

for all $k$. By applying the previous result to the sequence of blown-up functions $v_{k}$, we then obtain the microscopic description of blow-up asserted in Theorem 1.2 The characterization of the limit function $\psi$ follows from Theorem 2.4 .

\section{An example}

We demonstrate the absence of quantization also in the radially symmetric case by means of the following example.

Example 3.1. Consider the radially symmetric function $\varphi$ with

$$
\Delta^{2} \varphi=e^{-|x|^{2} / 2} \quad \text { in } \mathbb{R}^{4}, \quad \varphi(0)=\Delta \varphi(0)=0 .
$$

This function can be computed explicitly. In fact, for any $x \in \mathbb{R}^{4}$ we have

$$
\varphi(x)=\int_{0}^{|x|} s^{-3}\left\{\int_{0}^{s} t^{3}\left[\int_{0}^{t} \sigma^{-3}\left(\int_{0}^{\sigma} \tau^{3} e^{-\tau^{2} / 2} d \tau\right) d \sigma\right] d t\right\} d s .
$$

For $k \in \mathbb{N}$ and $x \in \mathbb{R}^{4}$ let

$$
u_{k}(x)=\ln k-\frac{k^{6}|x|^{2}}{8}+k^{-8} \varphi\left(k^{3} x\right) .
$$

Then $\left(u_{k}\right)$ satisfies equation (1), that is,

$$
\Delta^{2} u_{k}=V_{k} e^{4 u_{k}},
$$

where

$$
V_{k}(x)=e^{-4 k^{-8} \varphi\left(k^{3} x\right)} \rightarrow 1 \quad \text { in } C_{\mathrm{loc}}^{0}\left(\mathbb{R}^{4}\right) \text { as } k \rightarrow \infty .
$$

Thus, also (2) is satisfied. Finally, we compute that $V_{k} e^{4 u_{k}} \rightarrow 0$ in the sense of measures when $k \rightarrow \infty$.

Acknowledgements. The first author gratefully acknowledges the support of the Forschungsinstitut für Mathematik during his stay at ETH Zurich. 


\section{References}

[1] Adimurthi, Druet, O.: Blow-up analysis in dimension 2 and a sharp form of Trudinger-Moser inequality. Comm. Partial Differential Equations 29, 295-322 (2004) Zbl pre02130236 MR 2038154

[2] Adimurthi, Struwe, M.: Global compactness properties of semilinear elliptic equations with critical exponential growth. J. Funct. Anal. 175, 125-167 (2000) Zbl 0956.35045 MR 1774854

[3] Brézis, H., Merle, F.: Uniform estimates and blow-up behaviour for solutions of $-\Delta u=$ $V(x) e^{u}$ in two dimensions. Comm. Partial Differential Equations 16, 1223-1253 (1991) Zbl 0746.35006 MR 1132783

[4] Chang, S.-Y. A., Chen, W.: A note on a class of higher order conformally covariant equations. Discrete Contin. Dynam. Systems 7, 275-281 (2001) Zbl 1014.35025 MR 1808400

[5] Chen, W., Li, C.: Classification of solutions of some nonlinear elliptic equations. Duke Math. J. 63, 615-623 (1991) Zbl 0768.35025 MR 1121147

[6] Chen, X. X.: Remarks on the existence of branch bubbles on the blowup analysis of equation $-\Delta u=e^{2 u}$ in dimension two. Comm. Anal. Geom. 7, 295-302 (1999) Zbl 0928.35051 MR 1685598

[7] Druet, O.: Multibumps analysis in dimension 2-Quantification of blow up levels. Duke Math. J., to appear

[8] Druet, O., Robert, F.: Bubbling phenomena for fourth-order four-dimensional PDEs with exponential growth. Proc. Amer. Math. Soc., to appear

[9] Evans, L. C.: Partial Differential Equations. Grad. Stud. in Math. 19, Amer. Math. Soc. (1998) Zbl 0902.35002 MR 1625845

[10] Hebey, E., Robert, F., Wen, Y.: Compactness and global estimates for a fourth order equation of critical Sobolev growth arising from conformal geometry. Comm. Contemp. Math., to appear

[11] Li, Y., Shafrir, I.: Blow-up analysis for solutions of $-\Delta u=V e^{u}$ in dimension two. Indiana Univ. Math. J. 43, 1255-1270 (1994) Zbl 0842.35011 MR 1322618

[12] Lin, C. S.: A classification of solutions of a conformally invariant fourth order equation in $\mathbb{R}^{n}$. Comment. Math. Helv. 73, 206-231 (1998) Zbl 0933.35057 MR 1611691

[13] Malchiodi, A.: Compactness of solutions to some geometric fourth-order equations. J. Reine Angew. Math., to appear

[14] Malchiodi, A., Struwe, M.: The $Q$-curvature flow on $S^{4}$. J. Differential Geom., to appear

[15] Nicolescu, M.: Les fonctions polyharmoniques. Hermann, Paris (1936)

[16] Pizzetti, P.: Sulla media dei valori che una funzione dei punti dello spazio assume alla superficie di una sfera, Atti Accad. Naz. Lincei Cl. Sci. Fis., Mat. Nat. (5) 18, 182-185 (1909)

[17] Robert, F.: Positive solutions for a fourth order equation invariant under isometries. Proc. Amer. Math. Soc. 131, 1423-1431 (2003) Zbl pre01861494 MR 1949872

[18] Robert, F.: Quantization effects for a fourth order equation of exponential growth in dimension four. Preprint (2005)

[19] Robert, F., Struwe, M.: Asymptotic profile for a fourth order PDE with critical exponential growth in dimension four. Adv. Nonlinear Stud. 4, 397-415 (2004) Zbl pre02149271 MR 2100905

[20] Schoen, R. M.: Analytic aspects of the harmonic map problem. In: Seminar on Nonlinear Partial Differential Equations (Berkeley, CA, 1983), S. S. Chern (ed.), Springer, 321-358 (1984) Zbl 0551.58011 Zbl 0551.58011

[21] Wei, J.: Asymptotic behavior of a nonlinear fourth order eigenvalue problem. Comm. Partial Differential Equations 21, 1451-1467 (1996) Zbl 0872.35013 MR 1410837 